\title{
Optimal Strategies for the Control of Cholera with Three Infection Routes
}

\author{
Iddrisu A. Mohammed Katali ${ }^{*} \quad$ Baba Seidu $^{2} \quad$ Elijah B. Baloba ${ }^{2}$ \\ 1.Department of Mathematics, School of Engineering University for Development Studies, Tamale, Ghana, \\ P. O. Box 1350 \\ 2. Department of Mathematics, Faculty of Mathematical Sciences, C. K. Tedam University of Technology and \\ Applied Sciences, Navrongo, Ghana, UK-0215-2153
}

\begin{abstract}
.
Cholera is an acute intestinal infection and water borne disease that has claimed and continue to claim the lives of millions of people in the developing countries. Its effects on developing countries cannot be ignored since most of the people who get infected or die from the disease are usually children and adults who are in their economically active years. This paper presents an epidemic mathematical model that is aimed at describing the dynamics of the spread of Cholera. In this paper, infection of the disease is considered to be through contact with Infectives or vibrio cholerae in the dry lands and the water bodies. The model is qualitatively analyzed to determine conditions for successful fight against cholera. It is shown that if the basic reproduction number $\mathcal{R}_{0}$, is less than unity, then the disease-free equilibrium is locally asymptotically stable and the disease dies out and when the basic reproduction number is greater than unity, the vibrio cholerae and the disease will persist in the population. Numerical simulations are also carried out and various combinations of intervention strategies are compared to determine the most effective strategy that should be employed in order to control the spread of the v.cholerae. It is observed that the strategy that adopts education on open defecation, environmental sanitation and water treatment are the most effective strategies in the fight against the spread of the disease
\end{abstract}

Keywords and phrases: Mathematical model, local stability, basic reproduction number, optimal control.

DOI: $10.7176 / \mathrm{MTM} / 12-1-02$

Publication date: January $31^{\text {st }} 2022$

\section{Introduction}

Infectious diseases place next only to natural disasters in terms of threat to human race. Infectious diseases are disorders caused by organisms such as bacteria, viruses, fungi or parasites. Many of these organisms live in and on our bodies which are normally harmless or even helpful but under certain conditions some may cause diseases. The outbreak of infectious diseases such as tuberculosis, HIV, hepatitis, cholera and most recently Ebola, etc. causes loss of lives of millions of people in the human population as well as increases the expenditure of enormous amount of money in health care and disease control. It is therefore very important for us all to pay adequate attention to stopping the occurrence and the spread of such diseases by using effective controls and preventive measures. Many infectious diseases are as a direct result of poor hygienic conditions and contact between an infectious person and a susceptible person. Some infectious diseases are through blood like HIV, airborne viruses like tuberculosis and others are waterborne diseases like cholera.

Cholera is an acute bacterial infection of the intestine caused by the ingestion of food or water contaminated by certain strains of the Vibrio cholera organism [2]. The V. cholera produces enterotoxins whose actions on the mucosal epithelium are responsible for the characteristic symptoms of the disease, namely acute watery diarrhea and vomiting [1]. In the most severe cases, cholera is one of the most rapidly fatal illnesses known, due to severe dehydration [3]. It remains an important global cause of morbidity and mortality, capable of causing periodic epidemic disease [7]

Cholera spreads through contaminated food or drinking-water, as well as by person-to-person contact through the facial-oral route. Sanitary conditions in the environment play an important role since the V.cholerae bacterium survives and multiplies outside the human body and can spread rapidly where living conditions such as overcrowding, unprotected water sources and areas where there are no safe disposal of faeces. These conditions are mostly seen in poor countries, underdeveloped areas and in many refugee camps [3].

The cholera disease remains a global threat to public health and a key indicator of lack of social development. Recently, the reemergence of cholera has been noted in parallel with the ever-increasing size of vulnerable populations living in unsanitary conditions [7]. Mathematical modeling is an important tool used in analyzing the dynamics of infectious diseases [5]. Mathematical models help in understanding transmission of infections and assessing the potential impact of control strategies in reducing death and morbidity [1]. The following mathematical models on the cholera disease have been propose in literature to help improve the understanding of the transmission dynamics of the disease.

A simple deterministic model was developed by Codeco [11] to examine the role of aquatic reservoirs in the persistence of endemic cholera. This was done by the use of a Susceptible, Infected, Recovered (SIR) model 
incorporating aquatic population of Vibrio cholerae. Three hypothetical communities were used to illustrate the dynamics, these includes the endemic, epidemic and cholera free populations. Qualitative results of the cholera free population shows that the disease can be minimized by preventing water contamination, drinking of untreated water and by diluting cholera diarrhea using large quantities of water. The results of the model shown that the importance of the aquatic reservoir is dependent on the sanitary conditions of a community and that the rate of cholera reproduction is a product of social and environmental factors.

Mukandavire et al. [17] constructed a mathematical model which studied the 2008- 2009 cholera outbreak in Zimbabwe. In his model, he explores the "fast" human - to-human and "slow" environment-to-human transmission modes of cholera. His results show that both modes of transmission contributed in sustaining cholera outbreaks in Zimbabwe and that prevention of the outbreaks can be done through mass vaccination with a cholera vaccine that has moderate uptake. However, the model does not include the use of media as a tool for disseminating information on the availability of these vaccines.

Wang [9] extended Mukandavire et al. [17] to study environment to-human and human-to-human transmission model by incorporating three types of controls: vaccination, therapeutic treatment, and water sanitation. Their results show that different controls (such as vaccination and treatment) closely interplay with each other, and the specific population settings and relative costs determine the length and strength of each control in an optimal strategy.

In the work of Ochoche [19] a mathematical model for the control of cholera transmission dynamics using water treatment as a control strategy was proposed. The model is built on the assumption that cholera is contracted only through the ingestion of contaminated water. Their results showed that water treatment is an effective method of controlling cholera.

Edward and Nyerere [7] formulated a mathematical model that captured some essential dynamics of cholera transmission with public health educational campaigns, vaccination, sanitation and treatment as control strategies in limiting the disease. The reproduction numbers with single and combined controls were computed and compared with each other to assess the possible community benefits in the model. Their results show that treatment of the infective population yielded the best results followed by education campaign.

Onyuma et al. [21] extended the model proposed by Mukandavire et al. [17] to include the effects of media coverage in their work, they formulated a mathematical model for cholera transmission by incorporating media coverage. Their results shows that in the presence of media coverage the disease dies out faster while lack of effective media reporting on the presence of the disease and preventive measures greatly increases the number of infectious people in the population which is not favorable for the eradication of the disease.

This work seeks to investigate the mode of spread of the organism in the dry land and the water bodies in an epidemic model (SEIR) and incorporate optimal control in the form of education on open defecation, (environmental and water sanitation and personal hygiene) and treatment as control measures to curb the spread of the virus. The rest of the paper is organized as follows: a brief discussion of cholera is given in section 1 . In section 2 we formulated the mathematical model which consist of set of six nonlinear differential equations. A qualitative analysis of the model carried out which consist of positivity, boundedness of the model the equilibria of the model and local stability of the model was done in section 3. In section 4 we carry out sensitivity analysis of the model while optimal control theory is done in section 5 where we incorporate our control measures. Numerical simulations and conclusion are carried out in section 6 and 7 respectively.

\section{Model Formulation}

In this section, a mathematical model is proposed for the spread of cholera disease in a human population through contact with an infective person or ingestion of contaminated food or water with the vibrio cholera. Here, the total human population is divided at time $t$ into four mutually exclusive subpopulation such as Susceptible individuals (S), Exposed individuals (E), Infected individuals (I), and Recovered individuals (R). The bacterial population is also divided into two mutually exclusive subpopulations namely Vibrio cholerae in the dry lands $V_{d}$ : this class consist the El Tor biotype of v.cholerae 01 and v. cholerae 0139 Bengal in the dry lands. Vibrio cholerae in water bodies $V_{w}$ : this class includes all the v. cholerae 01 and $\mathrm{v}$. cholerae 0139 in the water bodies. The rate at which susceptible individual acquire infection is given by $\eta=\frac{\beta\left(E+I+V_{d}+V_{w}\right)}{1+\kappa}$, Where $\beta$ is the transmission contact rate for susceptible individuals with $E, I, V_{d}$ and $V_{w}$ respectively and $\kappa$ is the concentration of v. cholerae in food and water. After susceptible individuals become infected with $\mathrm{v}$. cholerae either through the dry lands, water bodies or an infected person, they have a portion joining the exposed class at $g$ and the remaining $(1-g)$ joining the infected class. Both the Exposed and the Infected individuals increases due to inflow of infectives from the susceptible population. Some individuals from the Exposed class moves to the Recovered at rate $\delta$ due to natural recovery, while a rate $\delta_{1}$ from the exposed class join the Infected class. Those individuals in the infected class can join the recovered class at a rate of $\lambda$ due to treatment or by natural recovery. The rate $\mu$ is the natural death rate of the human population, whereas $q$ is the disease induce death rate. $\gamma$ is the natural recruitment rate of vibrio cholerae in 
both the dry lands and the water bodies. It is assumed that the population of the bacteria concentration in both the dry lands and the water bodies increases at a rate of $\alpha$ and $\alpha_{1}$ respectively due to shading of bacteria from the exposed class.

The population of the v.cholera in both the dry lands and the water bodies also increases at a rates $\omega$ and $\omega_{1}$ respectively due to the relative shading bacteria by the infected class. The v. cholera in both the dry lands and the water bodies decreases due to natural death at a rate $\mu_{1}$ and also decreases at a rate $d$ due to the effect of both environmental and water disinfection. Some of the v. cholerae in the dry lands are washed to the water bodies at a rate $\varepsilon$ by running water from rains. Table 1 summarizes the definition of parameters and the baseline values used for numerical purpose only. Also, Figure 2.1 present the flow diagram of the model under discussion. Table 1. Description of Model Parameters and their baseline values.

\begin{tabular}{|l|l|l|l|}
\hline Par. & Description & Value & Ref. \\
\hline$\pi$ & Constant human recruitment rate & 0.99 & Assumed \\
\hline$\mu$ & Natural death rate of human population & 0.022 & {$[3]$} \\
\hline$q$ & Disease induced death rate & 0.052 & Assumed \\
\hline$\delta$ & Natural recovery rate of the exposed individuals & 0.003 & Assumed \\
\hline$\delta_{1}$ & Rate at which the exposed individuals get infected & 0.02 & Estimated \\
\hline$\lambda$ & Recovery rate of the infected individuals & 0.2 & {$[12]$} \\
\hline$\theta$ & Rate at which recovered individuals become susceptible & 0.001 & {$[13]$} \\
\hline$\mu_{1}$ & Natural death rate of the vibrio cholerae & 0.003 & Estimated \\
\hline$d$ & Death rate of vibrio cholerae due to treatment and sanitation & 0.033 & {$[13]$} \\
\hline$\beta$ & infection rate & 0.00025 & Assumed \\
\hline$\kappa$ & Vibrio cholerae infectious concentration & 100000 & {$[11]$} \\
\hline$g$ & Proportion of the susceptible who get exposed & 0.00035 & Assumed \\
\hline$\gamma$ & Natural recruitment rate of the vibrio cholerae & 0.85 & Assumed \\
\hline$\alpha$ & Rate of shedding v. cholerae by the exposed in to the dry lands & 0.009 & Assumed \\
\hline$\alpha_{1}$ & Rate of shedding v.cholerae by the exposed to the water bodies & 0.75 & Assumed \\
\hline$\omega$ & Rate of shedding v.cholerae by the infected to the dry lands & 0.006 & Assumed \\
\hline$\omega_{1}$ & Rate of shedding v. cholerae by the infected to the water bodies & 0.004 & Assumed \\
\hline$\varepsilon$ & Rate of v. cholerae that moves from dry lands to water bodies & 0.023 & Assumed \\
\hline
\end{tabular}

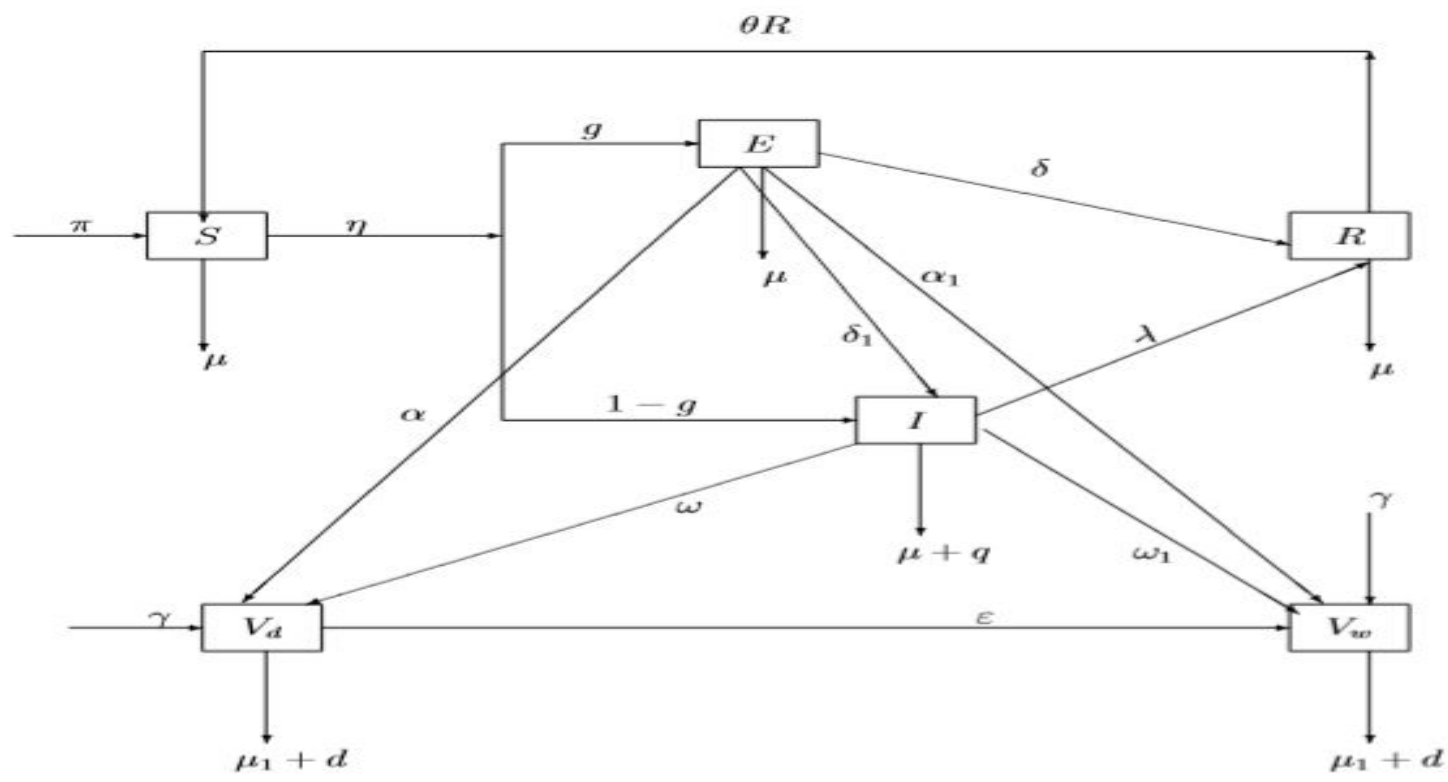

Figure 1. Schematic Diagram of cholera epidemic model in Human Populations

The model described above is therefore given by the following system of non-linear differential equations. 


$$
\left\{\begin{array}{c}
\frac{d S}{d t}=\pi+\theta R-(\eta+\mu) S \\
\frac{d E}{d t}=g \eta S-\left[\delta+\delta_{1}+\mu\right] E \\
\frac{d I}{d t}=(1-g) \eta S+\delta_{1}-[\lambda+\mu+q] I \\
\frac{d R}{d t}=\delta E+\lambda I-[\theta+\mu] R \\
\frac{d V_{d}}{d t}=\gamma+[\alpha E+\omega I]-\left[\mu_{1}+d+\varepsilon\right] V_{d} \\
\frac{d V_{w}}{d t}=\gamma+\left[\alpha_{1} E+\omega_{1} I+\varepsilon V_{d}\right]-\left[\mu_{1}+d\right] V_{w}
\end{array}\right.
$$

with positive initial conditions. In the next section, qualitative properties of the model are discussed.

\section{Qualitative Analysis}

\subsection{Positivity of Solutions.}

Since all the state variables in model (1) measure population or concentration, it is necessary that all such variables remain non-negative for all time $t$. This is given in the following.

Theorem 1. Given that the initial conditions of the system are non-negative, the future solutions are also nonnegative.

Proof. By [26], let $t>0$ be the first time such that $S(t)=0$. Then, from the first equation

$\frac{d S}{d t}=\pi+\theta R \geq 0$. Hence, $S(t) \geq 0, \forall t>0$. Similarly, for $\mathrm{t}>0$ then $\mathrm{E}(\mathrm{t})=0$, from the second equation $\frac{d E}{d t}=$ $g \eta S \geq 0$, for this reason $\mathrm{E}(\mathrm{t}) \geq 0, \forall t>0$. Similar arguments can be used to show that the other variables are non-negative, completing the proof.

\subsection{Boundedness of the Model.}

Since the model is dealing with population, we have to show that $S(t)>0, E(t)>0, I(t)>0, R(t)>0, V_{d}(t)>0$ and $V_{w}(t)>0 \forall t \in \mathbb{R}_{+}^{6}$. Which is stated in the following theorem.

Theorem 2. All solutions of the model are uniformly bounded and contained in the feasible region defined by $\Omega=$ $\Omega_{1} \times \Omega_{2} \forall t>0$, where $\Omega_{1}=\left\{(S(t), E(t), I(t), R(t)) \in \mathbb{R}_{+}^{4} \mid \lim _{t \rightarrow \infty} \operatorname{Sup} N(t) \leq \frac{\pi}{\mu}\right\} \quad$ and $\Omega_{2}=$ $\left\{\left(V_{d}(t), V_{w}(t)\right) \in \mathbb{R}_{+}^{2} \mid \lim _{t \rightarrow \infty} \operatorname{Sup} V(t) \leq \Gamma_{1}\right\}$, Where $\Gamma_{1}=\frac{2 \gamma \mu+\pi\left(\alpha+\alpha_{1}+\omega+\omega_{1}\right)}{\mu\left(\mu_{1}+d\right)}$. Thus, the region, $\Omega$ is positively invariant under the flow defined by model (1).

Proof. Consider the total human population as $N$ and the total bacterial population as $V$.

Then $N=S+E+I+R$, so that, $\frac{d N}{d t}=\pi-\mu N-q I$.

Using standard comparison theorems [27], [26], it can be shown that $\mathrm{N}(\mathrm{t}) \leq \frac{\pi}{\mu}+\left(N(0)+\frac{\pi}{\mu}\right) e^{-\mu t}$, so that $\lim _{t \rightarrow \infty} \operatorname{Sup} N(t) \leq \frac{\pi}{\mu}$.

Also, if $\mathrm{V}=V_{d}+V_{w}$ then it is easy to show that $\mathrm{V}(\mathrm{t}) \leq \Gamma_{1}$ where $\Gamma_{1}=\frac{2 \gamma \mu+\pi\left(\alpha+\alpha_{1}+\omega+\omega_{1}\right)}{\mu\left(\mu_{1}+d\right)}$.

Thus, that all solutions are uniformly bounded as given in the following definitions.

$\Omega_{1}=\left\{(S(t), E(t), I(t), R(t)) \in \mathbb{R}_{+}^{4} \mid \lim _{t \rightarrow \infty} \operatorname{Sup} N(t) \leq \frac{\pi}{\mu}\right\} \quad$ and $\quad \Omega_{2}=\left\{\left(V_{d}(t), V_{w}(t)\right) \in\right.$ $\left.\mathbb{R}_{+}^{2} \mid \lim _{t \rightarrow \infty} \operatorname{Sup} V(t) \leq \Gamma_{1}\right\}$

Therefore, the model (1) can conveniently study in $\Omega$, since $\Omega_{*}=\Omega_{1} \times \Omega_{2}$ is positively invariant under the model (1). This concludes the proof.

\subsection{Equilibria of the Model.}

The model can be shown to have a disease-free equilibrium given by $\mathcal{E}_{0}=\left(\frac{\pi}{\mu}, 0,0,0,0,0\right)$ and an endemic equilibrium $\mathcal{E}^{*}=\left(S^{*}, E^{*}, I^{*}, R^{*}, V_{d}^{*}, V_{w}^{*}\right)$. Were 


$$
\begin{gathered}
\left\{\begin{array}{c}
S^{*}=\frac{\theta R^{*}+\pi}{\eta^{*}+\mu}, \\
E^{*}=\frac{z_{1} \eta^{*}\left(\theta R^{*}+\pi\right)}{\eta^{*}+\mu}, \\
I^{*}=\frac{z_{2} \eta^{*}\left(\theta R^{*}+\pi\right)}{\eta^{*}+\mu}, \\
R^{*}=\frac{z_{3} \pi \eta^{*}}{\eta^{*}\left(1-\theta \zeta_{3}\right)+\mu}, \\
V_{d}^{*}=z_{4}+\frac{z_{5} \eta^{*}\left(\theta R^{*}+\pi\right)}{\eta^{*}+\mu} \text { and }, \\
V_{w}^{*}=\frac{1}{\mu_{1}+d}\left[\frac{\eta^{*}\left(\theta R^{*}+\pi\right)}{\eta^{*}+\mu}\left(\varepsilon z_{5}+\alpha_{1} z_{1}+\omega_{1} z_{2}\right)+\varepsilon Z_{4}+\gamma\right] \\
z_{1}=\frac{g}{\delta+\delta_{1}+\mu}, \\
z_{2}=\frac{(\mu+\delta)(1-g)+\delta_{1}}{\left(\delta+\delta_{1}+\mu\right)(\lambda+\mu+q)}, \\
z_{3}=\frac{((1-g) \lambda+\delta g) \mu+\left(\delta+\delta_{1}\right) \lambda+\delta g q}{\left(\delta+\delta_{1}+\mu\right)(\lambda+\mu+q)(\theta+\mu)}, \\
z_{4}=\frac{\gamma}{\mu_{1}+d+\varepsilon}, \\
z_{5}=\frac{\alpha z_{1}+\omega z_{2}}{\mu_{1}+d+\varepsilon}, \\
z_{6}=z_{2}+z_{5}+z_{1}+\frac{\varepsilon z_{5}+\alpha_{1} z_{1}+\omega_{1} z_{2}}{\mu_{1}+d} \text { and }, \\
z_{7}=z_{4}+\frac{\varepsilon z_{4}+\gamma}{\mu_{1}+d} .
\end{array}\right.
\end{gathered}
$$

And $\eta^{*}$ can be shown to satisfy the following cubic equation.

$\left(\eta^{*}\right)^{3}+Z_{2}\left(\eta^{*}\right)^{2}+Z_{1} \eta^{*}+Z_{0}=0$

Were

$Z_{2}=\frac{\mu}{\left(1-\theta z_{3}\right)}-\frac{\beta\left(\pi z_{6}+z_{7}\left(1-\theta z_{3}\right)\right)}{(1+\kappa)\left(1-\theta z_{3}\right)}+\mu ;$

$Z_{1}=\mu\left[\frac{\mu}{\left(1-\theta z_{3}\right)}-\frac{\beta\left(\pi z_{6}+z_{7}\left(1-\theta z_{3}\right)+z_{7}\right)}{(1+\kappa)\left(1-\theta z_{3}\right)}\right]$ and

$Z_{0}=-\frac{\beta z_{7} \mu^{2}}{(1+\kappa)\left(1-\theta z_{3}\right)}$

It is easy to verify that $1-\theta z_{3}>0$ and hence $Z_{0}<0$. Therefore, using the Descartes' rule of signs, the number of endemic equilibria of model (1) is determined by only $Z_{1}$ and $Z_{2}$ as follows.

i) There exists a unique endemic equilibrium whenever $Z_{1}$ and $Z_{2}$ have the same sign.

ii) There are three endemic equilibria whenever $Z_{1}>0$ and $Z_{2}<0$.

It can be seen that whenever $Z_{1}>0$, then $Z_{2} \nless 0$. The following result is therefore established.

Theorem 3.3. The Cholera model (1) exhibits a unique endemic equilibrium.

3.4. Local stability of disease-free Equilibrium.

The Jacobian of model (1) evaluated at $\varepsilon_{0}$ is given by

$$
\mathrm{J}\left(\varepsilon_{0}\right)=\left[\begin{array}{cccccc}
-\mu & -\frac{\pi \beta}{(1+\kappa) \mu} & -\frac{\pi \beta}{(1+\kappa) \mu} & \theta & -\frac{\pi \beta}{(1+\kappa) \mu} & -\frac{\pi \beta}{(1+\kappa) \mu} \\
0 & \frac{g \beta \pi}{(1+\kappa) \mu}-k_{1} & \frac{g \beta \pi}{(1+\kappa) \mu} & 0 & \frac{g \beta \pi}{(1+\kappa) \mu} & \frac{g \beta \pi}{(1+\kappa) \mu} \\
0 & \frac{(1-g) \beta \pi}{(1+\kappa) \mu}+\delta_{1} & \frac{(1-g) \beta \pi}{(1+\kappa) \mu}-k_{1} & 0 & \frac{(1-g) \beta \pi}{(1+\kappa) \mu} & \frac{(1-g) \beta \pi}{(1+\kappa) \mu} \\
0 & \delta & \lambda & -k_{5} & 0 & 0 \\
0 & \alpha & \omega & 0 & -k_{3} & 0 \\
0 & \alpha_{1} & \omega_{1} & 0 & \varepsilon & -k_{4}
\end{array}\right]
$$

Were

$k_{1}=\delta+\delta_{1}+\mu, k_{2}=\lambda+\mu+q, k_{3}=\mu_{1}+d+\varepsilon, k_{4}=\mu_{1}+d$ and $k_{5}=\theta+\mu$

Now, $\mathrm{J}\left(\varepsilon_{0}\right)$ can be shown to have two negative eigenvalues, $-\mu$ and $-k_{5}$, which are clearly negative and the remaining eigenvalues can be shown to satisfy the following characteristic polynomial equation.

Were

$$
X^{4}+\mathbb{A}_{3} X^{3}+\mathbb{A}_{2} X^{2}+\mathbb{A}_{1} X+\mathbb{A}_{0}=0
$$

$$
\mathbb{A}_{3}=k_{1}+k_{2}+k_{3}+k_{4}-\frac{\beta \pi}{(1+\kappa) \mu}
$$




$$
\begin{aligned}
& \mathbb{A}_{2}=\left(k_{3}+k_{4}+k_{2}\right) k_{1}+\left(k_{3}+k_{4}\right) k_{2}+k_{3} k_{4} \\
& -\frac{\beta\left(g\left(\alpha+\alpha_{1}+\delta_{1}+k_{2}\right)+k_{3}+k_{4}+\left(k_{1}+\omega_{1}+\omega\right)(1-g)\right) \pi}{(1+\kappa) \mu} \\
& \begin{array}{r}
\mathbb{A}_{1}=\left(\left(k_{3}+k_{4}\right)\right. \\
\left.k_{2}+k_{3} k_{4}\right) k_{1}+k_{3} k_{4} k_{1} \\
-\frac{\beta \pi}{\mu(1+\kappa)}\left\{g\left[\left(k_{3}+k_{4}+\omega_{1}+\omega\right) \delta_{1}+\left(\alpha+k_{3}+k_{4}+\alpha_{1}\right) k_{2}+k_{3} \alpha_{1}+\alpha\left(k_{4}+\varepsilon\right)\right]\right. \\
\left.+(1-g)\left[\left(k_{3}+k_{4}+\omega_{1}+\omega\right) k_{1}+\omega\left(k_{4}+\varepsilon\right)+k_{3} \omega_{1}\right]+k_{3} k_{4}\right\}
\end{array} \\
& \begin{array}{l}
\mathbb{A}_{0} \\
=k_{1} k_{2} k_{3} k_{4}
\end{array} \\
& -\frac{\left.\beta \pi\left(\left(\left(\delta_{1}+k_{2}\right) k_{4}+\delta_{1} \omega_{1}+k_{2} \alpha_{1}\right) k_{3}+\left(k_{4}+\varepsilon\right)\left(\alpha k_{2}+\omega \delta_{1}\right)\right) g+\left(\left(k_{4}+\omega_{1}\right) k_{3}+\omega\left(k_{4}+\varepsilon\right)\right)(1-g) k_{1}\right)}{\mu(1+\kappa)}
\end{aligned}
$$

The disease-free equilibrium is locally asymptotically stable if all zeros of (4) are negative. From Routh Hurwitz criteria, the following result is established.

Theorem 3.4. The disease-free equilibrium is locally asymptotically stable if the

following conditions are satisfied.

$$
\mathbb{A}_{0}>0, \mathbb{A}_{3}>0, \mathbb{A}_{2}-\frac{\mathbb{A}_{1}}{\mathbb{A}_{3}}>0 \text { and } \mathbb{A}_{1}-\frac{\mathbb{A}_{0} \mathbb{A}_{3}}{\mathbb{A}_{2}-\frac{\mathbb{A}_{1}}{\mathbb{A}_{3}}}>0 \text {. }
$$

\section{Sensitivity Analysis of the Endemic Equilibrium}

Sensitivity Analysis is a method used to quantify uncertainty, and its objective is to identify critical inputs that determine model output [24]. Inputs are deemed critical if slight changes in their values lead to substantial changes in model output. Determining critical inputs of models is very important as it helps in determining the parameters that ought to be given the utmost attention in terms of data capture and precision. Also, sensitivity indices help to determine parameters that can be manipulated to achieve desired results. Since persistence of Cholera is represented by the existence of the endemic equilibrium point, we perform sensitivity analysis of the endemic

\begin{tabular}{|c|c|c|c|c|c|c|}
\hline Par & $S^{*}$ & $E^{*}$ & $I^{*}$ & $R^{*}$ & $V_{d}^{*}$ & $V_{w}^{*}$ \\
\hline$\pi$ & 1.0000 & 1.0000 & 1.0000 & 1.0000 & $2.216 \mathrm{e}-6$ & $2.104 \mathrm{e}-6$ \\
\hline$\mu$ & -0.1899 & -0.6788 & -0.2703 & -1.2270 & $-6.017 e-7$ & $-7.424 \mathrm{e}-7$ \\
\hline$q$ & -0.005214 & -0.005216 & -0.1950 & -0.1950 & $-4.307 e-7$ & $-3.296 e-7$ \\
\hline$\delta$ & $5.525 \mathrm{e}-7$ & -0.06667 & $-9.841 e-6$ & $2.0 e-5$ & $-4.925 \mathrm{e}-10$ & $-2.837 e-8$ \\
\hline$\delta_{1}$ & $2.1 \mathrm{e}-6$ & -0.4444 & $8.84 \mathrm{e}-5$ & $7.419 \mathrm{e}-5$ & $-2.943 e-9$ & $-1.889 \mathrm{e}-7$ \\
\hline$\lambda$ & 0.007427 & 0.007421 & -0.7225 & 0.2775 & $-1.596 e-6$ & $-1.21 \mathrm{e}-6$ \\
\hline$\theta$ & 0.02629 & 0.02629 & 0.02629 & -0.01719 & $5.824 \mathrm{e}-8$ & $5.531 \mathrm{e}-8$ \\
\hline$\mu_{1}$ & 0.06988 & -0.01345 & -0.01345 & -0.01345 & -0.05085 & -0.0976 \\
\hline d & 0.7687 & -0.1479 & -0.1479 & -0.1479 & -0.5593 & -1.074 \\
\hline$\beta$ & -0.8386 & 0.1614 & 0.1614 & 0.1614 & $3.576 \mathrm{e}-7$ & $3.396 \mathrm{e}-7$ \\
\hline$\kappa$ & 0.8386 & -0.1614 & -0.1614 & -0.1614 & $-3.576 e-7$ & $-3.396 e-7$ \\
\hline $\mathrm{g}$ & $-4.726 e-6$ & 1.0000 & -0.0001989 & -0.0001669 & $6.622 \mathrm{e}-9$ & $4.25 \mathrm{e}-7$ \\
\hline$\gamma$ & -0.8386 & 0.1614 & 0.1614 & 0.1614 & 1.0000 & 1.0000 \\
\hline$\alpha$ & $-2.961 \mathrm{e}-9$ & $5.698 \mathrm{e}-10$ & $5.698 \mathrm{e}-10$ & $5.698 \mathrm{e}-10$ & $7.061 \mathrm{e}-9$ & $1.981 \mathrm{e}-9$ \\
\hline$\alpha_{1}$ & $-2.467 e-7$ & $4.749 e-8$ & $4.749 \mathrm{e}-8$ & $4.749 \mathrm{e}-8$ & $1.052 \mathrm{e}-13$ & $4.234 \mathrm{e}-7$ \\
\hline$\omega$ & $-9.26 e-7$ & $1.782 \mathrm{e}-7$ & $1.782 \mathrm{e}-7$ & $1.782 \mathrm{e}-7$ & $2.208 \mathrm{e}-6$ & $6.194 \mathrm{e}-7$ \\
\hline$\omega_{1}$ & $-6.173 e-7$ & $1.188 \mathrm{e}-7$ & $1.188 \mathrm{e}-7$ & $1.188 \mathrm{e}-7$ & $2.632 \mathrm{e}-13$ & $1.059 \mathrm{e}-6$ \\
\hline$\varepsilon$ & 0.0000 & 0.0000 & 0.0000 & 0.0000 & -0.3898 & 0.1711 \\
\hline
\end{tabular}
equilibrium to small changes in model parameters using the normalized forward sensitivity index described in [25], [28]. The sensitivity indexes are evaluated at the baseline parameter values in Table presented in Table 1 and presented in Table 2 .

Parameters for which the sensitivity indexes of $E^{*}, I^{*}, V_{d}^{*}$ and $V_{w}^{*}$ are positive (negative) promote (disease) Cholera spread. Thus increasing $\lambda, \theta, \beta, g, \gamma, \alpha, \alpha_{1}, \omega$ and $\omega_{1}$ increases the exposure of people to Cholera, while increasing $\mu, q, \delta, \delta_{1}, \mu_{1}, d$ and $\kappa$ decreases the exposure of people to Cholera. Similar observations can be made about the other state variables at endemic equilibrium.

Table 2. Sensitivity of endemic Equilibrium $\mathcal{E}^{*}$ to the model parameters

\section{Optimal Control Theory}

In this section we study the impact of controls on the spread of Cholera. To do that, we introduce three controls in to the basic model (1): 
i. The efficiency of education on open deification that will reduce the introduction of $\mathrm{V}$. cholerae in both land and water bodies $v_{e}$.

ii. The efficiency of both environmental and water sanitation and personal hygiene $v_{s}$.

iii. The efficiency of drug therapy for treatment in preventing $\mathrm{V}$. cholerae $v_{t}$.

With these controls, the model becomes

$$
\left\{\begin{array}{c}
\frac{d S}{d t}=\pi+\theta R-\left(\left(1-v_{e}\right) \eta+\mu\right) S \\
\frac{d E}{d t}=g\left(1-v_{e}\right) \eta S-\left[\delta+\delta_{1}+\mu\right] E \\
\frac{d I}{d t}=(1-g)\left(1-v_{e}\right) \eta S+\delta_{1}-\left[\lambda+\mu+v_{t}+q\right] I \\
\frac{d R}{d t}=\delta E+\left(\lambda+v_{t}\right) I-[\theta+\mu] R \\
\frac{d V_{d}}{d t}=\gamma+[\alpha E+\omega I]\left(1-v_{s}\right)-\left[\mu_{1}+d+\varepsilon\right] V_{d} \\
\frac{d V_{w}}{d t}=\gamma+\left(1-v_{t}\right)\left[\alpha_{1} E+\omega_{1} I+\varepsilon V_{d}\right]-\left[\mu_{1}+d\right] V_{w}
\end{array}\right.
$$

The main goal in this section is to find the control set $\mathbf{u}(t)$ and its associated state variables $S(t) ; E(t) ; I(t) ; R(t)$; $V_{d}^{*}$ and $V_{w}^{*}$ that maximizes the number of Susceptibles and minimizes cost of the controls as describe by the objective functional given by:

$J\left(v_{e}, v_{s}, v_{t}\right)=\int_{0}^{t f}\left[S-\left(B_{1} v_{e}^{2}+B_{2} v_{s}^{2}+B_{3} v_{t}^{2}\right)\right] d t$

Here $v_{e}^{2}, v_{s}^{2}$ and $v_{t}^{2}$ represent the unit costs of the various controls and the positive constants $B_{1}, B_{2}$ and $B_{3}$ are relative weights attached to the controls. Here, the quadratic function is used for the cost as estimate for the nonlinear function based on the assumption that the costs take nonlinear form. Thus, the objective is to find an optimal control set $v_{e}^{*}, v_{s}^{*}$ and $v_{t}^{*}$ such that

$J\left(v_{e}^{*}, v_{s}^{*}, v_{t}^{*}\right)=\max _{x}\left\{J\left(v_{e}, v_{s}, v_{t}\right) \mid v_{e}, v_{s}, v_{t} \in \boldsymbol{v}\right\}$

Where $v=\left\{\left(v_{e}, v_{s}, v_{t}\right) \mid 0 \leq v_{e}(t) \leq 1,0 \leq v_{s}(t) \leq 1,0 \leq v_{t}(t) \leq 1\right.$, are measurable over $\left.\left[0, t_{f}\right]\right\}$

is the set of admissible controls. The Pontryagin's Maximum Principle [15] provide necessary conditions for optimality of the control problem. This principle converts (5) and (6) into a problem of point-wise minimization of a Hamiltonian, $H$, with respect to $v_{e}, v_{s}$ and $v_{t}$.

The Hamiltonian is defined by

$H=L+\lambda_{1} \frac{d S(t)}{d t}+\lambda_{2} \frac{d E(t)}{d t}+\lambda_{3} \frac{d I(t)}{d t}+\lambda_{4} \frac{d R(t)}{d t}+\lambda_{5} \frac{d V_{d}(t)}{d t}+\lambda_{6} \frac{d V_{w}(t)}{d t}$.

Where $L$ is the integrand of $J$ and $\lambda_{i}=1,2,3,4,5,6$ are the adjoint variables associated with the state variables. By applying Pontryagin's maximum principle, the existence result for the optimal control pairs, the following result is obtained.

Theorem 5.1. There exists an optimal control set of $v_{\mathrm{e}}^{*}, \mathrm{v}_{\mathrm{s}}^{*}, \mathrm{v}_{\mathrm{t}}^{*}$ and corresponding solution, $\left(\mathrm{S}^{*}, \mathrm{E}^{*}, \mathrm{I}^{*}, \mathrm{R}^{*}, V_{\mathrm{d}}^{*}, \mathrm{~V}_{\mathrm{w}}^{*}\right)$, that maximizes the objective function $\mathrm{J}\left(\mathrm{v}_{\mathrm{e}}, \mathrm{v}_{\mathrm{s}}, \mathrm{v}_{\mathrm{t}}\right)$ over $\mathrm{v}$.

Furthermore, there exist adjoint functions $\lambda_{1}, \lambda_{2}, \lambda_{3}, \lambda_{4}, \lambda_{5}$ and $\lambda_{6}$ satisfy the following equations.

$$
\left\{\begin{array}{c}
\frac{d \lambda_{1}(t)}{d t}=\lambda_{1} \mu+\left(1-v_{e}\right) \eta \lambda_{1}-g\left(1-v_{e}\right) \eta \lambda_{2}-(1-g)\left(1-v_{e}\right) \eta \lambda_{3} \\
\frac{d \lambda_{2}(t)}{d t}=\left(\delta+\delta_{1}+\mu\right) \lambda_{2}-\delta_{1} \lambda_{3}-\delta \lambda_{4}-\left(1-v_{s}\right) \alpha \lambda_{5}-\left(1-v_{t}\right) \alpha_{1} \lambda_{6} \\
\frac{d \lambda_{3}}{d t}=(\lambda+\mu+q) \lambda_{3}-\lambda \lambda_{4}-\left(1-v_{s}\right) \omega \lambda_{5}-\left(1-v_{t}\right) \omega_{1} \lambda_{6} \delta_{1} \\
\frac{d \lambda_{4}}{d t}=(\theta+\mu) \lambda_{4}-\theta \lambda_{1} \\
\frac{d \lambda_{5}}{d t}=\left(\mu_{1}+d+\varepsilon\right) \lambda_{5}-\eta\left(\lambda_{1}+\lambda_{2}+\lambda_{3}\right)-\left(1-v_{s}\right)\left(1-v_{t}\right) \varepsilon \lambda_{6} \\
\frac{d \lambda_{6}}{d t}=\left(\mu_{1}+d\right) \lambda_{6}-\eta\left(\lambda_{1}+\lambda_{2}+\lambda_{3}\right)
\end{array}\right.
$$

and the transversality conditions $\lambda_{i}\left(t_{f}\right)=0 \quad i=1,2,3,4,5,6$.

Furthermore, the optimal control set of $\left(v_{e}^{*}, v_{s}^{*}, v_{t}^{*}\right)$ is obtain as

$$
\begin{gathered}
v_{e}^{*}=\min \left\{\max \left(0, \frac{\eta S^{*}(t)\left(\lambda_{1}-\lambda_{3}-\lambda_{5}-\lambda_{6}\right)}{2 B_{1}}\right), 1\right\} \\
v_{s}^{*}=\min \left\{\max \left(0, \frac{-(\alpha+\omega) V_{d}^{*} \lambda_{5}}{2 B_{2}}\right), 1\right\}
\end{gathered}
$$




$$
v_{t}^{*}=\min \left\{\max \left(0, \frac{-\left(\alpha_{1}+\omega_{1} \varepsilon\right) V_{w}^{*}}{2 B_{3}}\right), 1\right\}
$$

Proof: The adjoint equations and the transversality conditions are obtained from Pontryagin's Maximum Principle (P M P). We differentiate the Hamiltonian with respect to the state variables $S(t), E(t), I(t), V_{d}(t)$ and $V_{w}(t)$ respectively. The adjoint equation is given as:

$$
\left\{\begin{array}{cr}
\frac{d \lambda_{1}(t)}{d t}=\lambda_{1} \mu+\left(1-v_{e}\right) \eta \lambda_{1}-g\left(1-v_{e}\right) \eta \lambda_{2}-(1-g)\left(1-v_{e}\right) \eta \lambda_{3} \\
\frac{d \lambda_{2}(t)}{d t}= & \left(\delta+\delta_{1}+\mu\right) \lambda_{2}-\delta_{1} \lambda_{3}-\delta \lambda_{4}-\left(1-v_{s}\right) \alpha \lambda_{5}-\left(1-v_{t}\right) \alpha_{1} \lambda_{6} \\
\frac{d \lambda_{3}}{d t}= & (\lambda+\mu+q) \lambda_{3}-\lambda \lambda_{4}-\left(1-v_{s}\right) \omega \lambda_{5}-\left(1-v_{t}\right) \omega_{1} \lambda_{6} \delta_{1} \\
\frac{d \lambda_{4}}{d t}= & (\theta+\mu) \lambda_{4}-\theta \lambda_{1} \\
\frac{d \lambda_{5}}{d t}= & \left(\mu_{1}+d+\varepsilon\right) \lambda_{5}-\eta\left(\lambda_{1}+\lambda_{2}+\lambda_{3}\right)-\left(1-v_{s}\right)\left(1-v_{t}\right) \varepsilon \lambda_{6} \\
\frac{d \lambda_{6}}{d t}= & \left(\mu_{1}+d\right) \lambda_{6}-\eta\left(\lambda_{1}+\lambda_{2}+\lambda_{3}\right)
\end{array}\right.
$$

Using the optimality conditions, we find

Hence, this gives as

$$
\begin{array}{cc}
\frac{\partial H}{\partial v_{e}}=-2 B_{1} v_{e}+\eta S^{*}(t) \lambda_{1}-\eta S^{*}(t) \lambda_{3}-\eta S^{*}(t) \lambda_{5}-\eta S^{*}(t) \lambda_{6}=0 & \text { at } v_{e}^{*} \\
\frac{\partial H}{\partial v_{s}}=-2 B_{2} v_{s}-(\alpha+\omega) V_{d}^{*} \lambda_{5}=0 \quad \text { at } v_{s}^{*} \\
\frac{\partial H}{\partial v_{t}}=-2 B_{3} v_{t}-\left(\alpha_{1}+\omega_{1}\right) V_{w}^{*} \lambda_{6}=0 \quad \text { at } v_{t}^{*}
\end{array}
$$

$$
\begin{aligned}
v_{e}^{*}(t) & =\frac{\eta S^{*}(t)\left(\lambda_{1}-\lambda_{3}-\lambda_{5}-\lambda_{6}\right)}{2 B_{1}} \\
v_{S}^{*}(t) & =\frac{-(\alpha+\omega) V_{d}^{*}(t) \lambda_{5}}{2 B_{2}} \\
v_{t}^{*}(t) & =\frac{-\left(\alpha_{1}+\omega_{1}\right) V_{W}^{*}(t) \lambda_{6}}{2 B_{3}}
\end{aligned}
$$

From control arguments involving bonds, the optimum control has the following form

$$
v_{e}^{*}= \begin{cases}\frac{\eta S^{*}(t)}{2 B_{1}}\left(\lambda_{1}-\lambda_{3}-\lambda_{5}-\lambda_{6}\right), & \text { if } 0<\frac{\eta S^{*}\left(\lambda_{1}-\lambda_{3}-\lambda_{5}-\lambda_{6}\right)}{2 B_{1}}<u_{e} \\ 0, & \text { if } \frac{\eta S^{*}(t)}{2 B_{1}}\left(\lambda_{1}-\lambda_{3}-\lambda_{5}-\lambda_{6}\right) \leq 0 \\ u_{e}, & \text { if } \frac{\eta S^{*}(t)}{2 B_{1}}\left(\lambda_{1}-\lambda_{3}-\lambda_{5}-\lambda_{6}\right) \geq u_{e}\end{cases}
$$

Also

$$
v_{s}^{*}=\left\{\begin{array}{lr}
\frac{-(\alpha+\omega) V_{d}^{*}(t) \lambda_{5}}{2 B_{2}}, & \text { if } 0<\frac{-(\alpha+\omega) V_{d}^{*}(t) \lambda_{5}}{2 B_{2}}<u_{s} \\
0, & \text { if } \frac{-(\alpha+\omega) V_{d}^{*}(t) \lambda_{5}}{2 B_{2}} \leq 0 \\
u_{s}, & \text { if } \frac{-(\alpha+\omega) V_{d}^{*}(t) \lambda_{5}}{2 B_{2}} \geq u_{s}
\end{array}\right.
$$

Similarly, we conclude

$$
\begin{aligned}
& v_{t}^{*}=\left\{\begin{array}{l}
\frac{-\left(\alpha_{1}+\omega_{1}\right) V_{w}^{*}(t) \lambda_{6}}{2 B_{3}}, \\
0, \\
u_{t},
\end{array}\right. \\
& \begin{array}{cc}
\text { if } 0<\frac{-\left(\alpha_{1}+\omega_{3}\right) V_{w}^{*}(t) \lambda_{6}}{2 B_{3}}< & u_{t} \\
\text { if } \frac{-\left(\alpha_{1}+\omega_{1}\right) V_{w}^{*}(t) \lambda_{6}}{2 B_{3}} & \leq 0 \\
\text { if } \frac{-\left(\alpha_{1}+\omega_{1}\right) V_{w}^{*}(t) \lambda_{6}}{2 B_{3}} & \geq u_{t}
\end{array}
\end{aligned}
$$

\subsection{Optimality of the System.}

The optimality system consists of the state system (5) coupled with the adjoint system (9) incorporating with the characterization of the optimal control set and initial and transversality conditions. Then we obtained the following optimality system: 


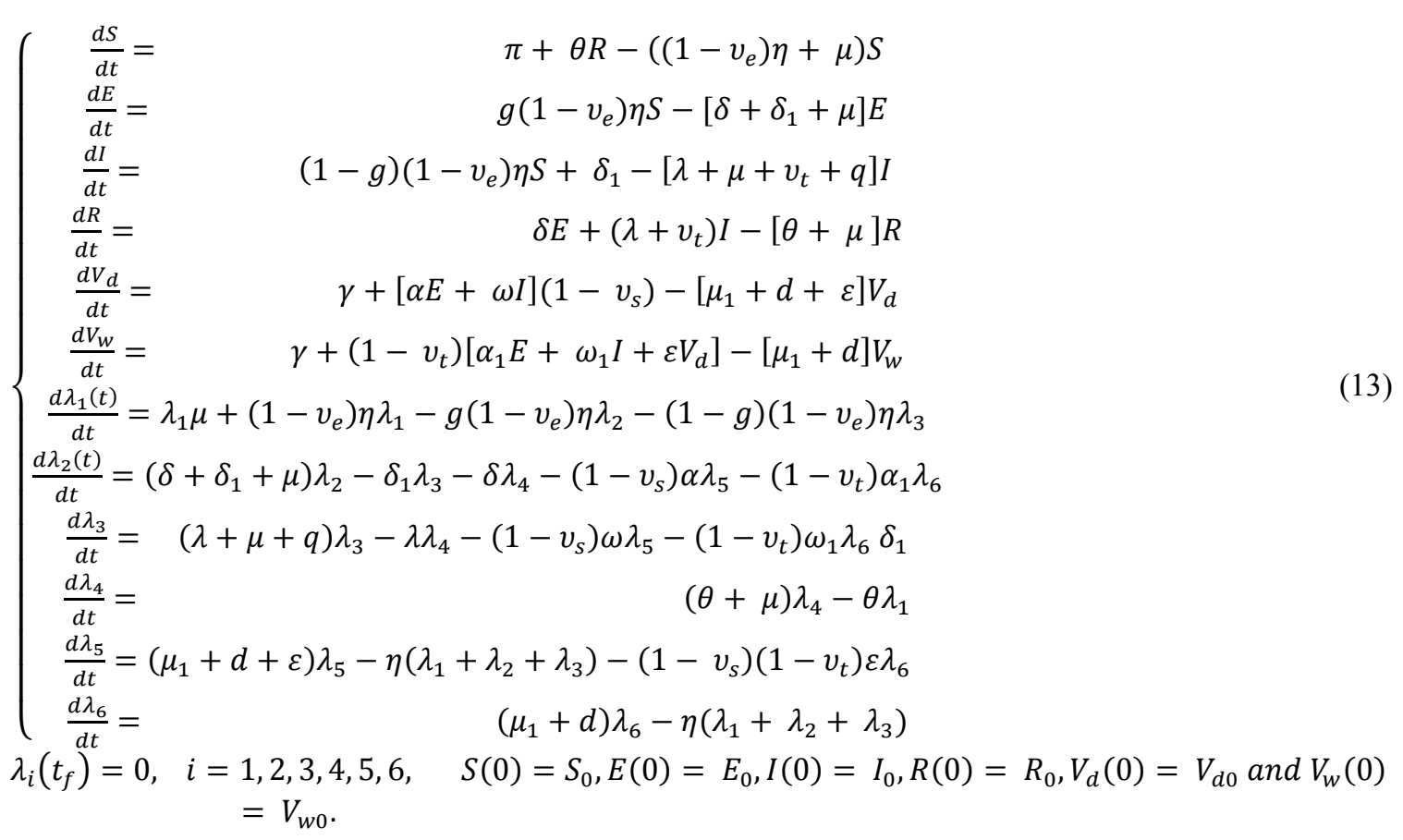

\section{Numerical Simulation}

In this section, we carry out numerical simulation to study the impact of parameters and controls on the spread of v. cholerae on the basic model (1) and the resulting optimality system consisting of the state equations (5) and the adjoint system (9). We make use of the parameter values given in Table 1.

An iterative scheme is used to find the optimal solution of the optimality system. Since the state system (5) has initial conditions and the adjoint system (9) has final conditions, the state system is solved using Runge-Kutta fourth order method in order to see the dynamical behavior of the system (5) in the presence of the control parameters. We first solve the state equation by forward Runge -Kutta fourth order method for the time $t_{f}=12$ months starting with the initial guess for the adjoint variables. Also due to the final condition of the adjoint system, we use a backward fourth order Runge Kutta method to solve it with the same time interval.

The simulations were performed using the initial conditions: $S(0)=60, E(0)=30, I(0)=20, R(0)=$ $40, V_{d}(0)=50$ and $V_{w}(0)=55$ and taking the time interval $[0,200]$ where $t_{0}=0$ and $t_{f}=12$ months.

To examine the impact of each control measure on the eradication of cholera disease, we consider the following as intervention

- Applying education on open defecation only $\left(v_{e}\right)$

- Applying sanitation only $\left(v_{s}\right)$

- Applying treatment only $\left(v_{t}\right)$

- Applying education on open defecation $\left(v_{e}\right)$ and sanitation $\left(v_{s}\right)$ only

- Applying education on open defecation $\left(v_{e}\right)$ and treatment $\left(v_{t}\right)$ only

- Applying sanitation $\left(v_{s}\right)$ and treatment $\left(v_{t}\right)$ only

- Applying all the three controls that is education on open defecation $\left(v_{e}\right)$, sanitation $\left(v_{s}\right)$ and treatment $\left(v_{t}\right)$

Using the above parameters values in table 1, a graph of the infectious population with respect to time in (months) has been drown. From the figure 2 it is seen that when there are no control measures, the spread of the v.cholerae increases in both the dry lands and the water bodies and letter maintain to a constant level and also result to an increase in the infected individuals. We conclude that there is the need to always put on preventive measures to stop the breading of the bacteria in the lands and the water bodies.

Again we simulated the model system by incorporating education on open defecation intervention only. Figure 3 shows a gradual decrease of the infectious population in the specified time. Hence, we can conclude that educating the people against open defecation is a vital control measure in reducing the spread bacteria and as well as cholera infections.

Again incorporating sanitation only on the model, it is seen from figure 4 that the exposed individuals and as well as the v.cholerae in the water bodies slightly decreases while the infected individuals and v.cholerae in the dry lands slightly decrease to a point and then decreases with the specified time. Also incorporating treatment only as a control measure, it can be seen in figure 5 shows that all the infected, exposed and v.cholerae population decreases but infected individuals and the bacteria in the water bodies did not get zero over the period of intervention. 
Again we simulated the model by pairing all the three control measures that is education on open defecation $\left(v_{e}\right)$, environmental and water sanitation $\left(v_{s}\right)$ and treatment $\left(v_{t}\right)$ and the graphs can be seen in figure 6 , figure 7 and figure 8 and in all the graphs there is a decrease in the infected and exposed individuals as well as a decrease in the v.cholerae population in both the dry lands and the water bodies.

Finally, we perform the simulation by incorporating all the control measures on the model to eradicate cholera and its associate agents in the population. Figure 9 shows that all the infected individuals, the v.cholerae population in the water bodies, the exposed individuals and that of the v.cholerae in the dry lands decreases to zero during the implementation period of the controls. Hence, we conclude that applying this controls effectively for a very long period will eradicate the disease and its associated agents from the community.

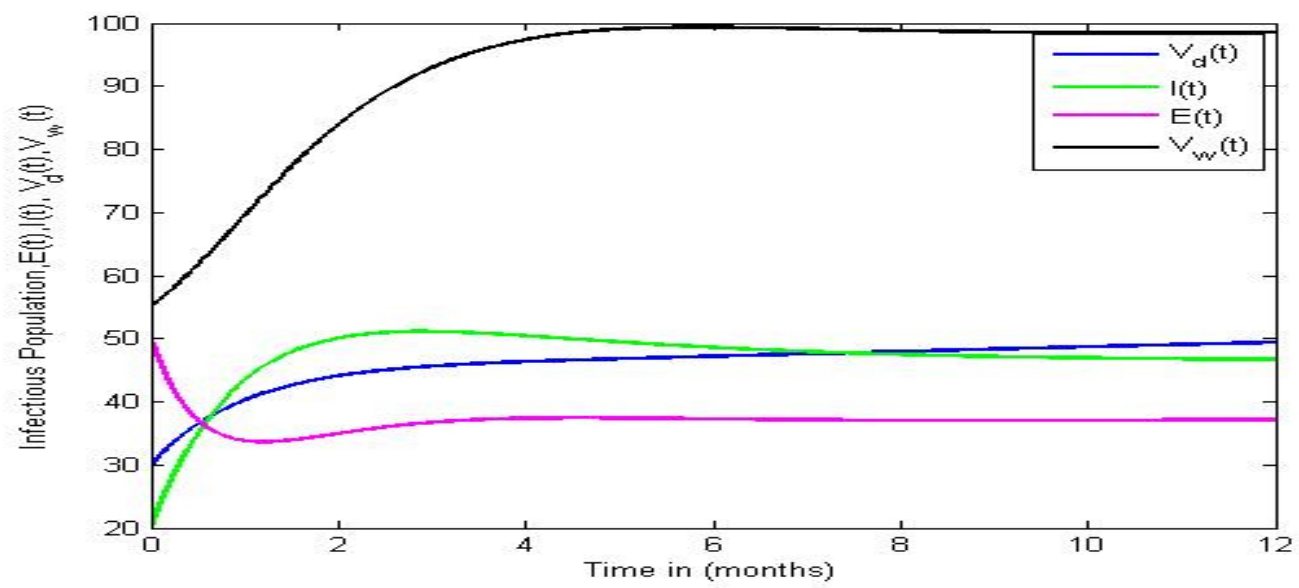

Figure 2: Simulation of model without control measures

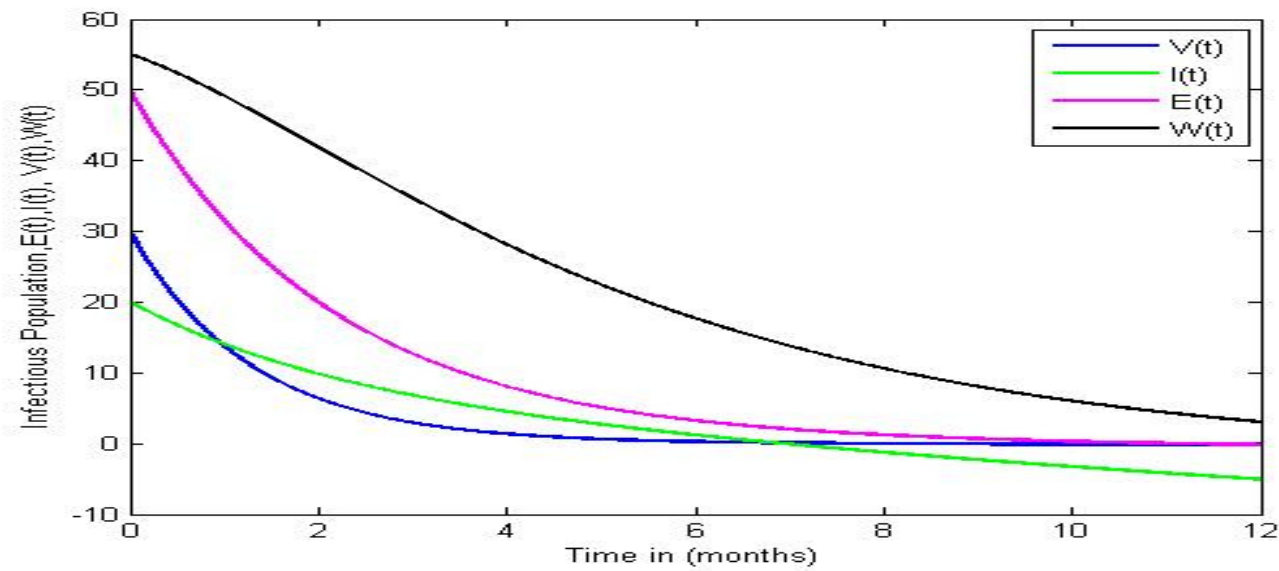

Figure 3: Impact of education on open defecation on infectious population

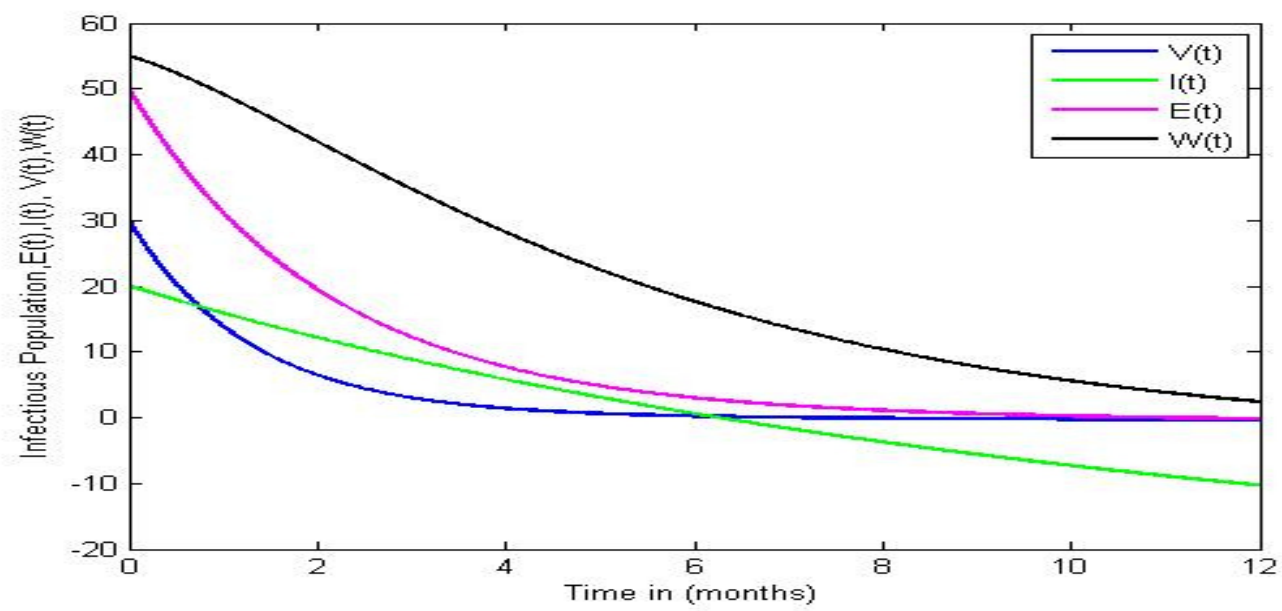

Figure 4: Impact of sanitation on infectious population 


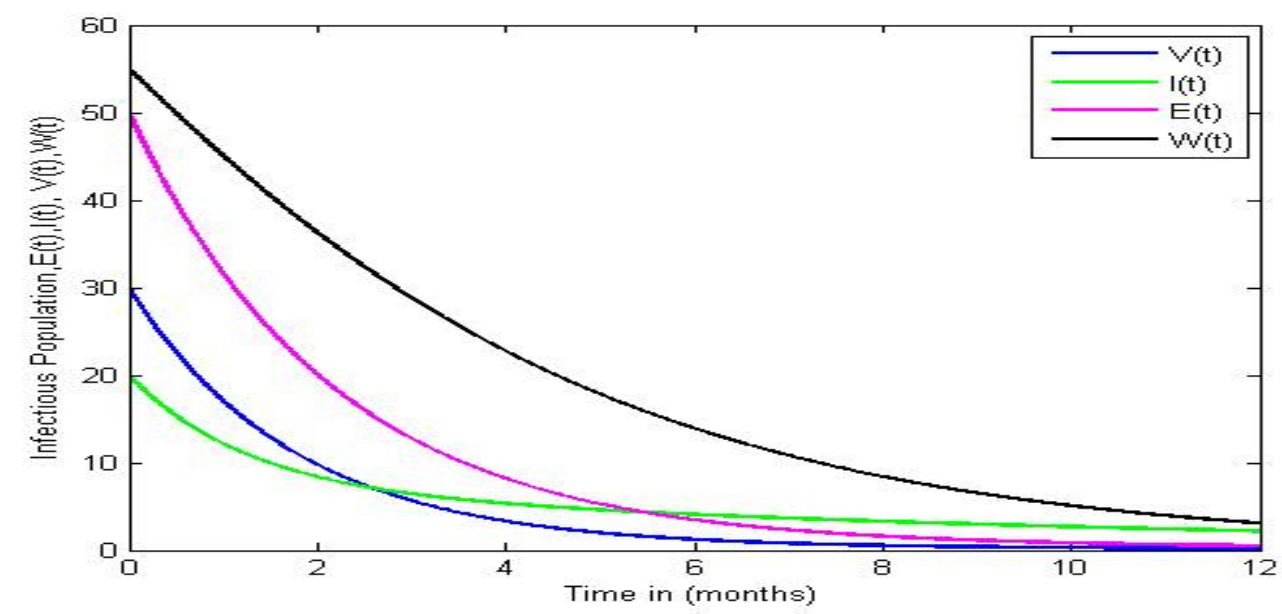

Figure 5: Impact of treatment only

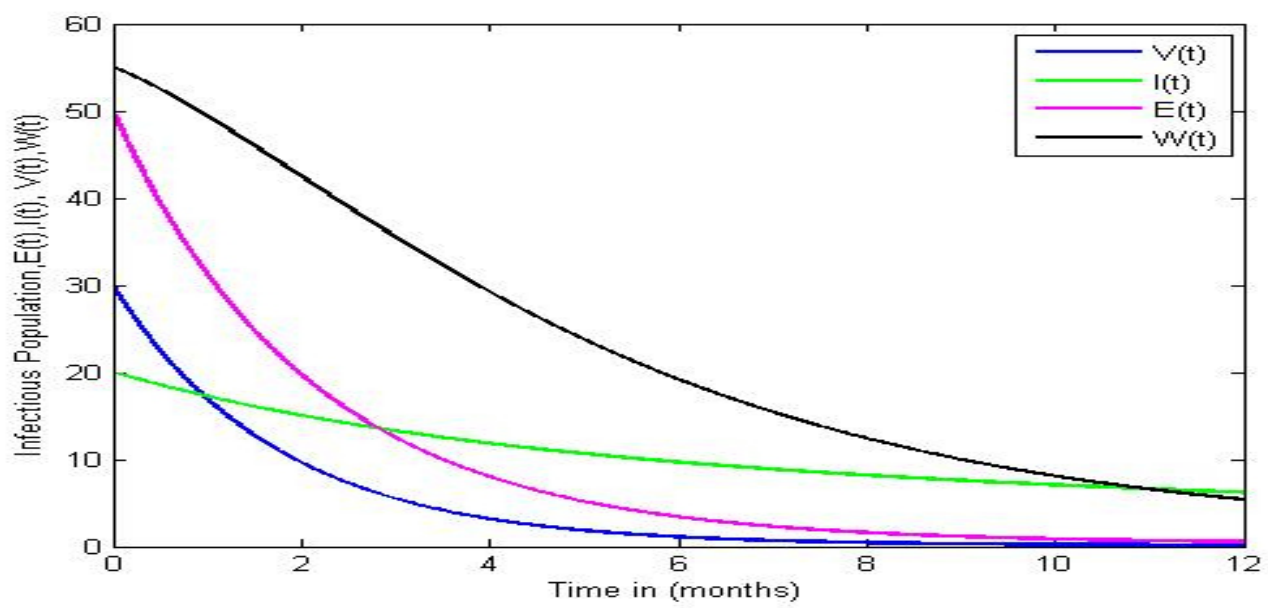

Figure 6: Simulation of model with open defecation and sanitation controls

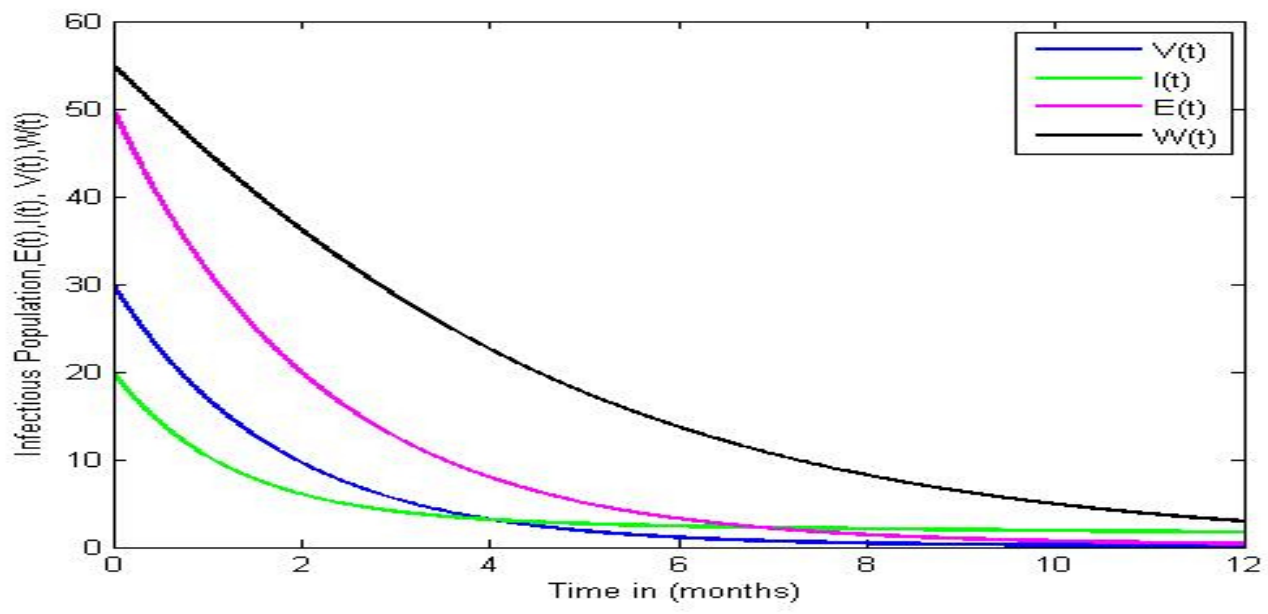

Figure 7: Simulation of model with open defecation and treatment controls 


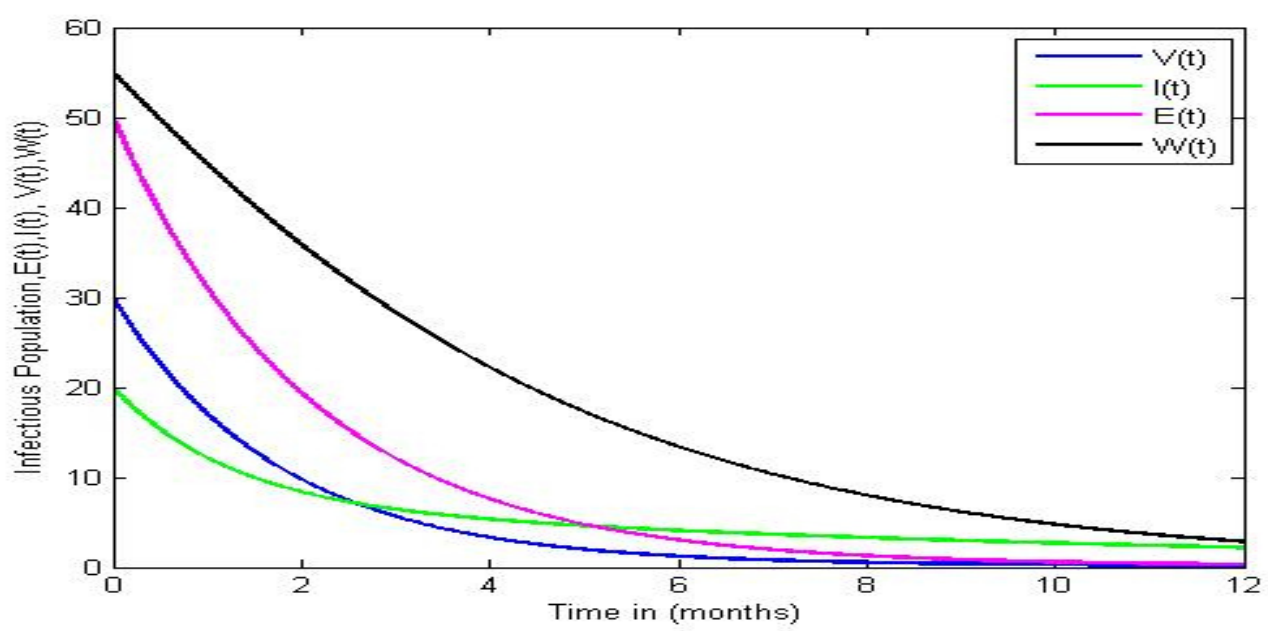

Figure 8: Simulation of model with sanitation and treatment controls

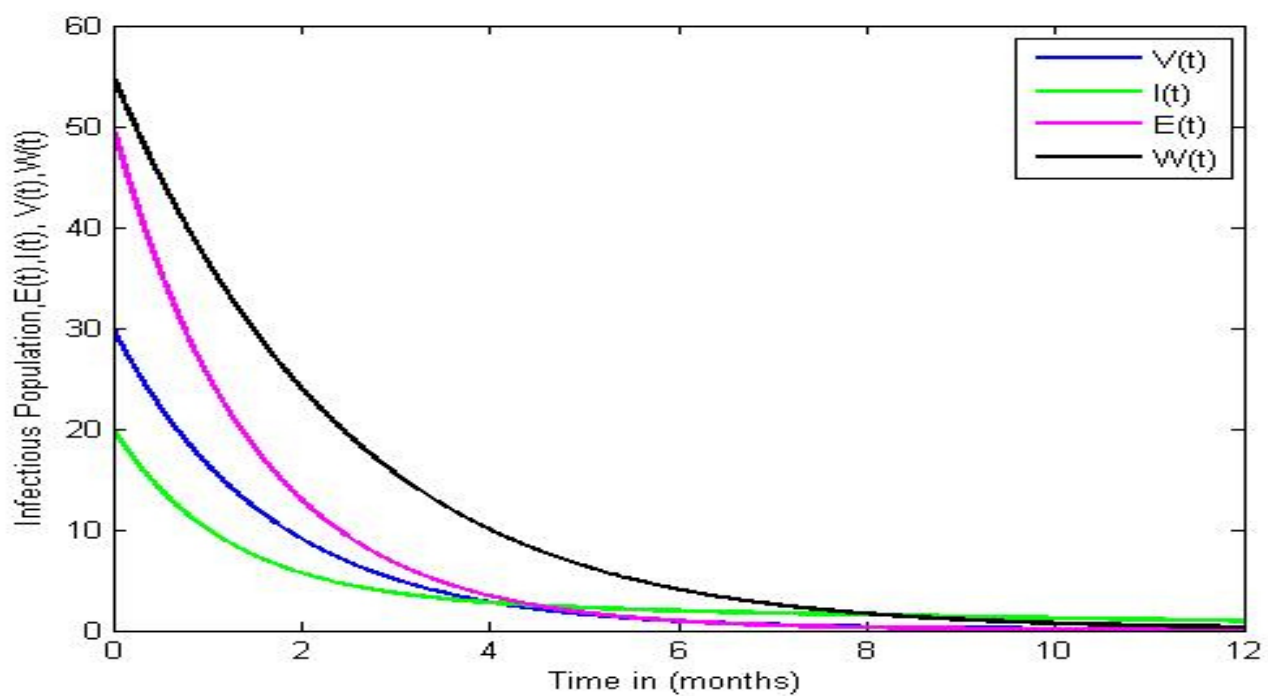

Figure 9: Simulation of model with all the three controls

\section{Conclusion}

In this study, a mathematical model based on a system of ordinary differential equations was used to examine the dynamical behavior of an epidemic of cholera disease considering relationship between the Susceptible human, Exposed human, Infected human, Recovered human and vibrio cholerae in dry lands and vibrio cholerae in water bodies has been formulated and analyzed. Analysis of the model shows that there exists a region where the model is mathematically and epidemiologically well posed because its solutions were positive and bounded. The basic reproduction number, which was the threshold parameter, was done using the next generation matrix approach. It was determined that when $\mathcal{R}_{0}<1$, cholera does not spread. Stability analysis of the cholera model shows that the disease-free equilibrium is locally asymptotically stable when the basic reproduction number is less than unity. Ideally, this means that keeping $\mathcal{R}_{0}$ less than unity is a possible strategy for curbing the spread of the disease. Analysis of the endemic equilibrium shows its existence when $\mathcal{R}_{0}>1$.

Sensitivity analysis of the model shows that a reduction in the rate of human-to-human transmission, environment to human transmission, rate of recruitment of susceptible and the rate of human shedding of Vibrio cholerae to the dry lands and the water bodies is required to reduce the basic reproduction number and the disease spread. On the other hand, education on open defecation and environmental sanitation will reduce the pathogen concentration required for a person to be infected with the disease, the rate of recovery, and the rate of death of Vibrio cholerae is required to reduce the basic reproduction number and to lower the spread of the disease. Optimal control problem is formulated and the conditions of optimal control of the disease are analyzed with effective preventive measures (education on open defecation, environmental sanitation and treatment). Existence and optimality system is also developed. The proposed prevention strategies are investigated numerically and their results are displayed graphically. The findings shows that lack of effective education on open defecation, both on 
land and water sanitation as a preventive measure greatly increases the number of people infected by cholera and the vibrio cholerae population.

Data Availability. The numerical data used to support the findings of this research are included within the article. Conflicts of Interest. The authors declare that there are no conflicts of interest regarding the publication of this paper.

\section{References}

[1] Lee, K. (2001). The Global Dimensions of Cholera. Global Change and Human Health, 2(1), 6-17.

[2] K. Alexander and T. Kirschner. Rapid Growth of Planktonic Vibrio Cholerae Non o1/Nono139 Strains in a Large Alkaline Lake in Austria: Dependence on Temperature and Dissolved Organic Carbon Quality. Applied and Environmental Microbiology, 74:2004-2015, 2008.

[3] World Health Organization. (2008). Prevention and control of cholera outbreaks: WHO policy and recommendations. WHO Regional Office for the Eastern Mediterranean, (November), 1-4.

[4] S. Edward, and N Nyerere 2015. A Mathematical Model for the Dynamics of Cholera with Control Measures Control Measures." ResearchGate 4(2): 53-63.

[5] H. W. Hethcote, "The mathematics of infectious diseases ", SIAM Review, vol 42, no. 4, pp599-653, 2000.

[6] Overview, C., \& Distribution, C. (2013). Cholera Epidemiology and Response. Unicef, 3-4.

[7] Edward, S., \& Nyerere, N. (2015). A Mathematical Model for the Dynamics of Cholera with Control Measures. (Http://Www.Sciencepublishinggroup.Com/j/Acm, 4(2), 53-63.

[8] Driessche, P. Van Den, \& Watmough, J. (2002). Reproduction numbers and sub-threshold endemic equilibria for compartmental models of disease transmission. Mathematical Biosciences, 180, 29-48.

[9] Wang, J. I. N., \& Modnak, C. (2011). Modeling Cholera Dynamics with Controls. Canadian Applied Mathematics Quarterly, 19(3), 255-274.

[10] Bertuzzo, E., Finger, F., Gatto, M., \& Rinaldo, A. (2016). On the probability of extinction of the Haiti cholera epidemic. Stochastic Environmental Research and Risk Assessment, 30(8), 2043-2055.

[11] Code'co, C. T. (2001). Endemic and epidemic dynamics of cholera: the role of the aquatic reservoir. BMC Infectious Diseases.

[12] Hartley, D. M., Jr, J. G. M., \& Smith, D. L. (2006). Hyper infectivity: A Critical Element in the Ability of V. cholerae to Cause Epidemics? PLoS Medicine www.Plosmedicine. Org, (May 2014).

[13] Panja, P., \& Kumar, S. (2014). A mathematical study on the spread of Cholera. South Asian Journal of Mathematics, 4(2), 69-84.

[14] Clark, R. N. (1992). The Routh-Hurwitz Stability Criterion, Revisited. (June), 3-4.

[15] L. S. Pontryagin, V. G. Boltyanskii, R. V. Gamkrelidze, and E. F. M. (1962). The Mathematical Theory of Optimal Processes (4th ed., Vol. 4).

[16] Levin, R. (2010). Vibrio cholerae. In Rapid Detection and Characterization of Foodborne Pathogens by Molecular Techniques (Seventh Ed).

[17] Mukandavire, Z., Liao, S., Wang, J., Gaff, H., Smith, D. L., \& Morris, J. G. (2009). Using mathematical models and R 0 to better understand epidemics: the 2008-2009 cholera outbreak in Zimbabwe.

[18] B. Seidu, O. D. Makinde, and I. Y. Seini, Mathematical Analysis of the Effects of HIV Malaria Co-Infection on Workplace Productivity, Acta Biotheoretica, vol. 63, no. 2, pp. 151 - 824, 2015.

[19] Ochoche, J. M. (2013). A Mathematical Model for the Transmission Dynamics of Cholera with Control Strategy. Nternational Journal of Science and Technology Volume, 2(11), 797-803.

[20] Abdulai Taofeek. (2015). Using Atanackovic and Stankovic Numerical Method to Investigate Fractional Order Cholera Mode. Kwame Nkrumah University of Science and Technology, Kumasi.

[21] Onyuma, M. B., Submitted, A. T., Partial, I. N., Of, F., For, R., Award, T. H. E., Sciences, P. (2017). Modelling cholera transmission incorporating media coverage.

[22] Havelaar, A. H., Protection, H., Jacob, J., \& Elster, B. (1997). Vibrio cholerae 1. 119-142.

[23] Gorrill, K. B. A. R. H. (1957). A Study of Antigenic Variation in Vibrio cholerae. 721-729.

[24] Marino, R. A., \& Levy, R. (2008). Spatial Relationships of Visuomotor Transformations in the Superior Colliculus Map. (September).

[25] Chitnis, N., Cushing, J. M., \& Hyman, J. M. (2006). Bifurcation Analysis of a Mathematical Model for Malaria Transmission. SIAM Journal on Applied Mathematics, 67(1), 24-45.

[26] Baloba B. E, Baba S. and Borna S. C. (2020). Mathematical Analysis of the Effects of Controls on the Transmission Dynamics of Anthrax in both Animal and Human Populations. Computational and Mathematical Methods in Medicine, Vol. 2020.

[27] V. Lakshmikanthan, S. Leela, and A. A. A. Martyniuk, Stability analysis of nonlinear systems, CRC Press, 1989

[28] C. S. Bornaa, B. Seidu and M. I. Daabo (2020). Mathematical Analysis of Rabies Infection. Journal of Applied Mathematics, Vol. 2020 